Document downloaded from:

http://hdl.handle.net/10251/54396

This paper must be cited as:

Sánchez Escuderos, D.; Ferrando Bataller, M.; Berenguer Verdú, AJ.; Baquero Escudero, M.; Valero-Nogueira, A. (2014). Dielectric Bed of Nails in Gap-Waveguide Technology at Millimeter-Wave Frequencies. IEEE Microwave and Wireless Components Letters. 24(8):515-517. doi:10.1109/LMWC.2014.2321496.

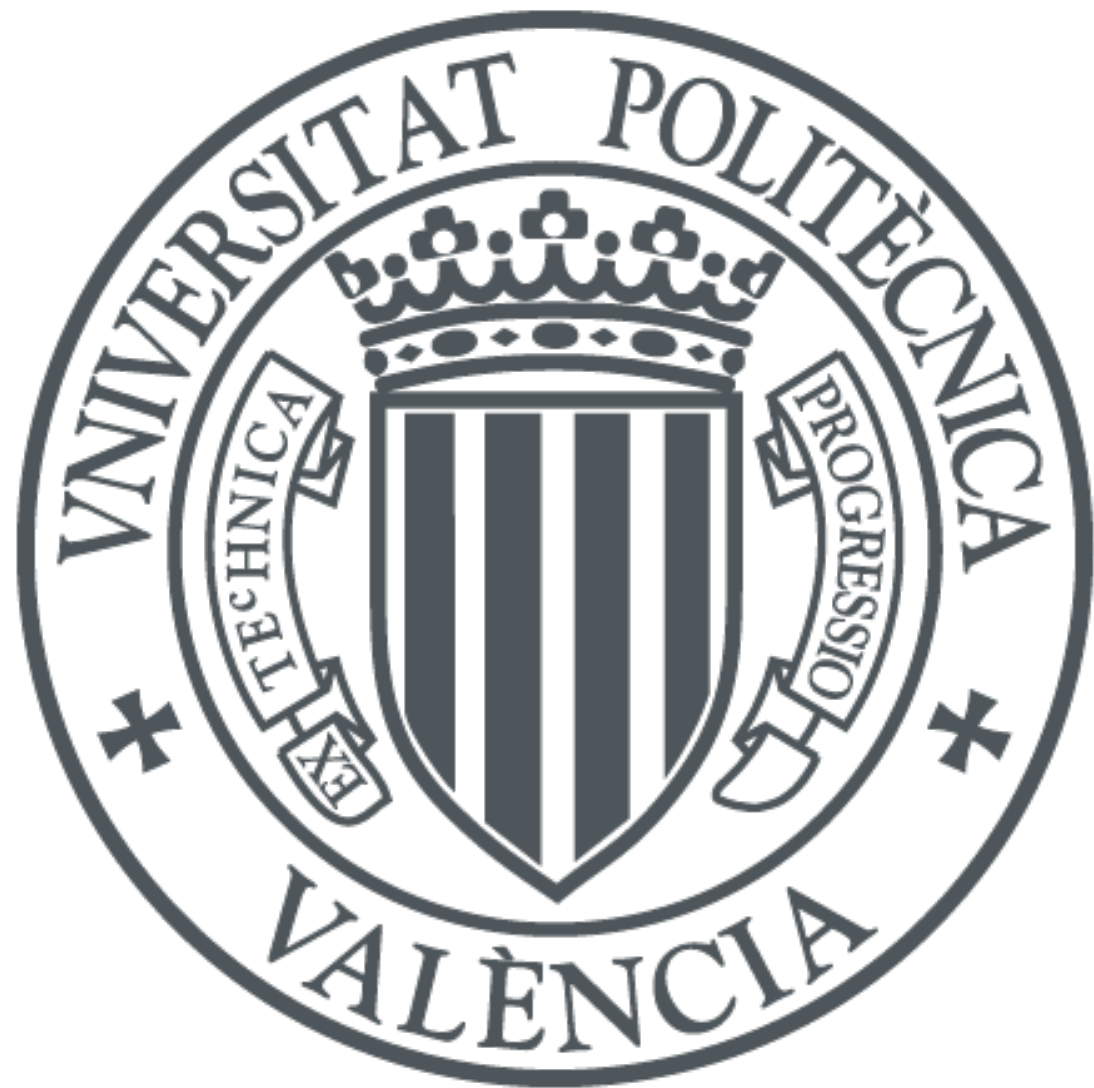

The final publication is available at

http://dx.doi.org/10.1109/LMWC.2014.2321496

Copyright Institute of Electrical and Electronics Engineers (IEEE)

Additional Information

"(c) 20xx IEEE. Personal use of this material is permitted. Permission from IEEE must be obtained for all other users, including reprinting/ republishing this material for advertising or promotional purposes, creating new collective works for resale or redistribution to servers or lists, or reuse of any copyrighted components of this work in other works." 


\title{
Dielectric Bed of Nails in Gap-Waveguide Technology at Millimeter-Wave Frequencies
}

\author{
Daniel Sánchez-Escuderos, Member, IEEE, Miguel Ferrando-Bataller, Member, IEEE, Antonio Berenguer, \\ Mariano Baquero-Escudero, Member, IEEE, and Alejandro Valero-Nogueira, Senior Member, IEEE,
}

\begin{abstract}
Gap-waveguide technology is based on the guidance of a quasi-TEM mode between two PEC plates. The confinement is possible due to a PEC/PMC condition on laterals, which prohibits the propagation of waves on those directions. PMC condition is commonly created by means of a metallic bed of nails. This letter examines the possibility of using a dielectric bed of nails to impose the PMC condition. The use of such kind of nails may facilitate the insertion of active components. Measurements for a straight transmission line and a power divider are included to verify the correct behavior of the dielectric bed of nails.
\end{abstract}

Index Terms-Gap-waveguide, Electromagnetic bandgap (EBG) structures, Dielectric EBG, millimeter-wave technology.

\section{INTRODUCTION}

$\mathbf{T}$ HE design of waveguides at millimeter and submillimeter wave frequencies entails additional problems, not present at lower frequencies. For instance, hollow metallic rectangular waveguides must have a perfect conducting metal joint to avoid losses on walls whereas microstrip lines are affected by high dielectric losses. To solve these problems, the gap-waveguide technology has been recently proposed [1].

Gap waveguides are based on the stopband produced in all directions between a PEC plate and a PMC plate separated less than $\lambda / 4$. Under this situation, if a PEC path is introduced in the PMC plate, the energy is confined between the PEC plate and the PEC path in the way of a quasi-TEM mode. In order to create a PMC plate, a high impedance surface implemented by means of a metallic bed of nails is commonly used [2]. The complete waveguide has been analytically studied in [3] and experimentally validated in [4].

The advantages of the gap-waveguide technology include low attenuation (the quasi-TEM mode propagates through air), and the possibility of having non-perfect metallic joints (PEC and PMC plates may be separated up to $\lambda / 4$ ). These advantages allow the design of low-loss transmission lines [4] and circuits with solid ridge [5] or suspended strips [6].

Gap waveguides may be fed with vertical [4] or longitudinal pins [6]. In case of suspended-strip gap waveguides, a transition from rectangular waveguide to metallic strip has been proposed [7] for measuring prototypes without soldering.

Manuscript received December 18, 2013; revised February 27, 2014; accepted April 19, 2014.

This work has been supported by the Spanish Ministry of Education and Science (Ministerio de Educacion y Ciencia) under the projects TEC201020841-C04-01 and CSD2008-00068

The authors are with the Instituto de Telecomunicaciones y Aplicaciones Multimedia (ITEAM) of the Universitat Politècnica de València (UPV), Cami de Vera s/n, 46022, Valencia, Spain (e-mail: dasanes1@iteam.upv.es).

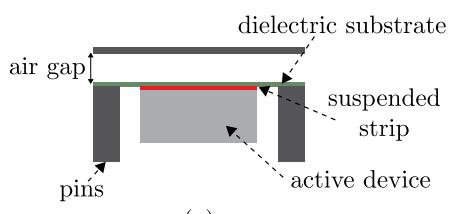

(a)

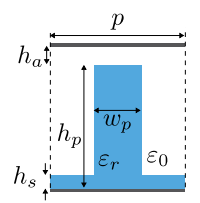

(b)

Fig. 1. Bed of nails: (a) insertion of active component and (b) unit cell of the dielectric bed of nails.

The inclusion of active components in gap-waveguide technology have not yet been addressed. Major difficulties arise from the height of the active component, which may be larger than the available air gap. For instance, the height of a commercial RF-MEMS of Radant MEMS working up to $40 \mathrm{GHz}$ is $0.65 \mathrm{~mm}$, whereas the required air gap in the low millimeter-wave band is $0.4 \mathrm{~mm}$.

An easy way to insert such switch consists in using a suspended-strip design and placing the switch between pins, as shown in Fig. 1 (a). If the feeding network of the RF-MEMS (formed by a $\lambda / 4$ line and a radial stub [8]) also lies on the bottom face of the dielectric, a short-circuit might be produced between the feeding network and the metallic bed of nails.

In order to avoid the above problem, this letter proposes the substitution of the metallic bed of nails by a dielectric bed of nails. By doing so, metallic strips may be located on both faces of the thin dielectric substrate without creating short-circuits with pins. To achieve a stopband, this kind of bed needs a high-permittivity dielectric, e.g. silicon. This material might be used at higher frequencies to directly implement an active component, e.g. a PIN diode, in the dielectric pins by creating doped regions with deposition techniques.

\section{Dielectric Bed Of NAILS}

A dielectric bed of nails must implement a high-impedance surface on the top of the pins which, in conjunction with the upper metallic plate, creates a stopband in the air gap that prohibits the propagation of waves in all directions. To do so, the air gap's height must be smaller than $\lambda / 4$ and the bed of nails must be shielded by a metallic plate in the bottom side.

The unit cell of the proposed dielectric bed of nails, shown in Fig. 1 (b), is formed by a square dielectric pin embedded in air. A thin dielectric floor $\left(h_{s}\right)$ is included for the ease of fabrication. Lower and upper metallic plates are indicated by dark lines.

The bandwidth of the stopband is determined by the dielectric: the higher the permittivity, the wider the stopband. 
TABLE I

MAIN CHARACTERISTICS OF DIFFERENT BED OF NAILS.

\begin{tabular}{c||c|c|c}
\hline Parameter & Metallic & $\begin{array}{c}\text { Dielectric } \\
\left(\text { RO3006, } \varepsilon_{r}=6.15\right)\end{array}$ & $\begin{array}{c}\text { Dielectric } \\
\left.\text { (TMM10i, } \varepsilon_{r}=9.9\right)\end{array}$ \\
\hline \hline$h_{p}(\mathrm{~mm})$ & 2.37 & 2.86 & 2.56 \\
\hline$w_{p}(\mathrm{~mm})$ & 1.02 & 1.17 & 1.05 \\
\hline$p(\mathrm{~mm})$ & 2.91 & 3.43 & 3.07 \\
\hline $\mathrm{B}(\%)$ & 71.84 & 1.83 & 17.34 \\
\hline$\alpha(\mathrm{dB} / \mathrm{cm})$ & 0.34 & 0.41 & 0.44 \\
\hline
\end{tabular}

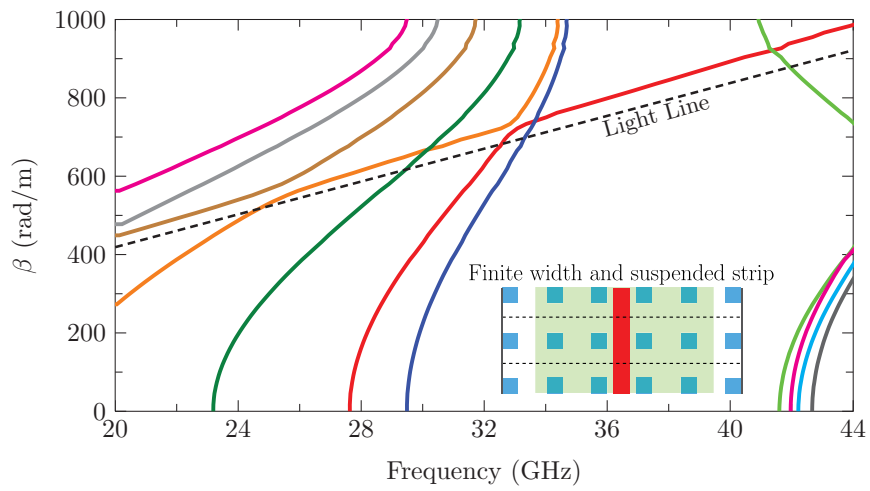

Fig. 2. Dispersion diagram of the dielectric bed of nails with suspended strip.

In the proposed design, a Rogers $\mathrm{TMM}^{\circledR}{ }^{\circledR} 10 \mathrm{i}$ substrate, with $\varepsilon_{r}=9.9$ and $\tan \delta=0.08$ in the Ka band, has been chosen. The unit cell has been optimized to maximize a stopband centered at $36.6 \mathrm{GHz}$. Table I compares the optimal dimensions considering $h_{a}=0.4 \mathrm{~mm}$ for the proposed dielectric, as well as a lower-permittivity dielectric and a metallic bed of nails.

The power transmission in the designed dielectric bed of nails is done by means of a suspended strip, which was recently proposed to test several circuits on the same bed of nails [6]. This configuration has been simulated with Ansys HFSS [9] considering a finite number of pins in the transverse direction ( 3 pins on each side of the strip ended in a metallic wall) and periodic boundary conditions in the longitudinal direction (see inset of Fig. 2). It has been assumed a goldplated copper metallic strip with a thickness $t=35 \mu \mathrm{m}$ and a width $w=0.95 \mathrm{~mm}$, suspended on Pyralux ${ }^{\circledR}$ AP-9121R of DuPont $^{T M}$, with $h=50.8 \mu \mathrm{m}, \varepsilon_{r}=3.6$ and $\tan \delta=0.06$.

Fig. 2 shows the dispersion diagram of the simulated structure with the stopband centered at $36.6 \mathrm{GHz}$. The inclusion of the suspended strip allows the propagation of a quasi-TEM mode in the stopband. Fig. 3 (a) shows the total electric field on the $x_{0}$ transverse plane (see Fig. 4 (a)) at $37 \mathrm{GHz}$. As can be seen, the energy is mainly propagated between the upper metallic plate and the metallic strip. Similar field distributions may be obtained at other frequencies within the stopband.

Table I shows the bandwidth (B) and attenuation $(\alpha)$ of the suspended-strip transmission line over the different bed of nails. As can be observed, a metallic bed of nails presents a larger bandwidth and less attenuation than a dielectric bed. However, the bandwidth of the high-permittivity dielectric bed of nails may be enough in many applications, specially if

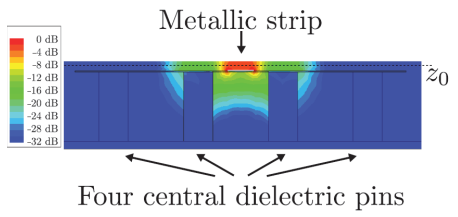

(a)

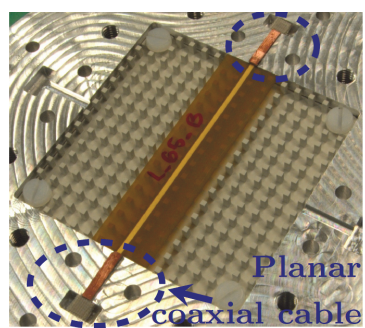

(b)
Fig. 3. Suspended strip over dielectric bed of nails: (a) Total electric field on a transverse plane passing through a pin row at $37 \mathrm{GHz}$ and (b) Picture of the manufactured prototype (upper metallic plate is not present).

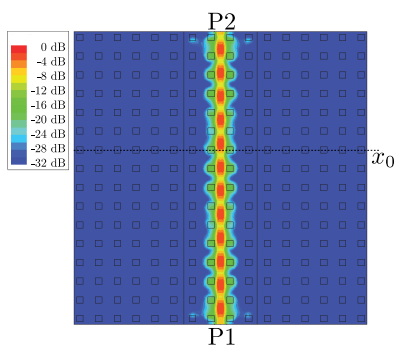

(a)

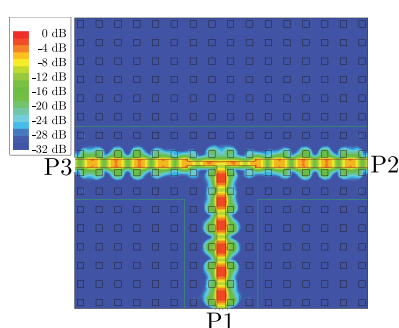

(b)
Fig. 4. Electric field on a longitudinal plane in the air gap at 37GHz: a) transmission line and $\mathrm{b}$ ) power divider.

active components with narrow-band feeding networks are used. Table I also shows that a lower-permittivity dielectric would produce a considerable bandwidth reduction.

\section{TRANSMISSION LINE AND POWER DIVIDER: MANUFACTURING AND MEASUREMENT}

The designed dielectric bed of nails has been manufactured in a Rogers $\mathrm{TMM}^{\circledR} 10 \mathrm{i}$ substrate. The stopband of this bed has been tested with a transmission line and a power divider.

Fig. 3 (b) shows a picture of the transmission line (suspended strip on a Piralux ${ }^{\circledR}$ substrate) over the $16 \times 16$ dielectric bed of nails. Feeding is done through the test fixture presented in [7]. This feeding uses a $50 \Omega$-planar coaxial cable (dashed circles in Fig. 3 (b)) formed by a central metallic strip separated from the upper and bottom planar ground planes by a PTFE substrate. On one end, the planar coaxial cable is fed by the input rectangular waveguide and, on the other end, the central metallic trip is connected to the suspended strip.

The impedance of the suspended-strip transmission line is determined by the width $w$ of the strip and the position with respect to the pins, as in a metallic bed of nails [6]. A strip with $w=1.4 \mathrm{~mm}$ and located between two rows of pins provides a $50 \Omega$ impedance. However, in order to reduce the amount of energy transmitted through the nearest row of dielectric pins, a narrower strip $(w=0.95 \mathrm{~mm})$ is chosen. Matching is deteriorated $(Z=65 \Omega)$ but preserved within acceptable values.

Fig. 4 (a) shows the total electric field along the transmission line of Fig. 3 (b) on $z_{0}$ plane (see Fig. 3 (a)) at $37 \mathrm{GHz}$. As can be seen, the quasi-TEM mode is not spread to the surrounding dielectric bed of nails along the complete metallic strip. 


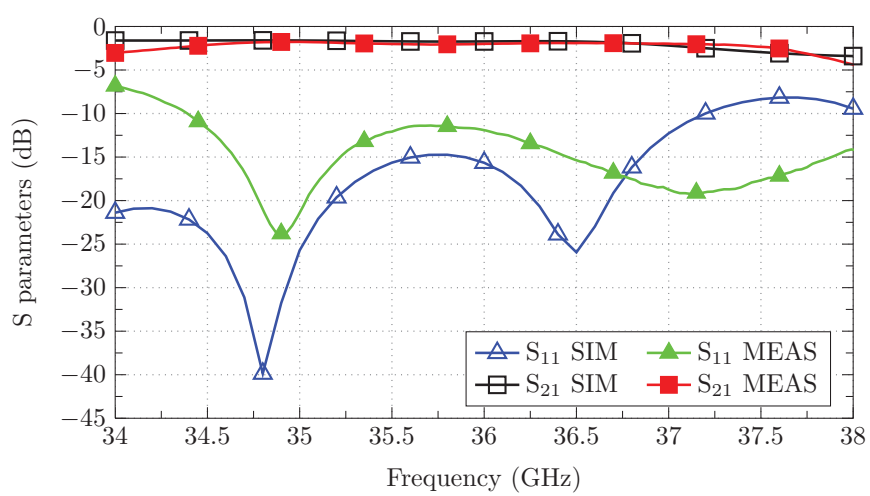

Fig. 5. Simulated and measured S-parameters of a $65 \Omega$ transmission line in suspended strip technology over a dielectric bed of nails.

The transmission line has been measured with WR28 transitions from 34 to $38 \mathrm{GHz}$. In order to obtain the S-parameters between points P1 and P2 in Fig. 4 (a), a TRL calibration has been applied using the TRL calibration kit described in [7]. Measured and simulated S-parameters are shown in Fig. 5. Simulations have also been corrected by a TRL calibration, simulating the THRU, REFLECT and LINE circuits.

Results of Fig. 5 show a good agreement between simulated and measured S-parameters. Despite a small increment in the $S_{11}$ parameter due to small assembling mismatches, the transmission line is matched within the stopband. Insertion loss is $2 \mathrm{~dB}$ from 35 to $37 \mathrm{GHz}$, which means an attenuation of $0.44 \mathrm{~dB} / \mathrm{cm}$ (note that, if $w=1.4 \mathrm{~mm}$, the attenuation would be $0.54 \mathrm{~dB} / \mathrm{cm}$ ). The same transmission line over a metallic bed of nails fabricated with aluminium (see Table I) presents an attenuation of $0.34 \mathrm{~dB} / \mathrm{cm}$. This difference is kept small because power is mainly transmitted through air in both cases.

The dielectric bed of nails has been also tested with a power divider. Fig. 4 (b) shows a diagram of the power divider over the bed of nails in conjunction with the position of the different ports. This figure also shows the total electric field along the metallic strip of the power divider in the air gap (on $z_{0}$ plane) at $37 \mathrm{GHz}$. As can be observed, the energy is not spread to the surrounding dielectric pins.

Fig. 6 shows the measured and simulated S-parameters of the power divider. As in previous example, a TRL calibration kit has been used in both, measured and simulated parameters, to obtain the S-parameters between ports P1, P2 and P3 in Fig. 4 (b). Results show a good matching in port P1 of the power divider. Measured insertion loss in ports $\mathrm{P} 2$ and $\mathrm{P} 3$ is, in average, $5 \mathrm{~dB}$. The excess of $2 \mathrm{~dB}$ in the insertion loss with regard to the ideal value $(3 \mathrm{~dB})$ is caused by the attenuation along the metallic strip shown in previous transmission line.

\section{CONCLUSIONS}

The proposed dielectric bed of nails is able to transmit a quasi-TEM mode in a suspended strip within the stopband of the structure. The bed of nails has been manufactured and tested with a transmission line and a power divider with a good agreement between measurements and simulations.

Since the energy is mainly transmitted through an air region, the dielectric bed of nails just introduces a small increment in

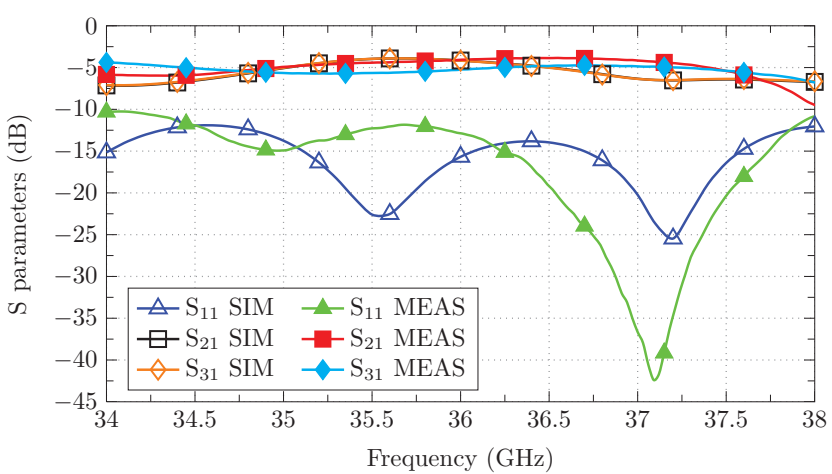

Fig. 6. Simulated and measured S-parameters of a power divider in suspended strip technology over a dielectric bed of nails.

the attenuation compared to a metallic bed of nails. In addition, the reduction of bandwidth (see Table I) with respect to a metallic bed of nails might be acceptable in many applications.

A dielectric bed of nails may be useful in circuits with metallic strips on the bottom face of the thin dielectric substrate. This possibility is essential when inserting active devices to avoid the short-circuiting of the feeding network and the pins. Moreover, the necessary high permittivity of the dielectric may be useful to directly implement an active component in the bed of nails at higher frequencies.

\section{REFERENCES}

[1] P.-S. Kildal, E. Alfonso, A. Valero-Nogueira, and E. Rajo-Iglesias, "Local metamaterial-based waveguides in gaps between parallel metal plates," IEEE Antennas and Wireless Propagation Letters, vol. 8, pp. 84-87, 2009.

[2] M. G. Silveirinha, C. A. Fernandes, and J. R. Costa, "Electromagnetic characterization of textured surfaces formed by metallic pins," IEEE Transactions on Antennas and Propagation, vol. 56, no. 2, pp. 405-415, 2008.

[3] A. Polemi, S. Maci, and P.-S. Kildal, "Dispersion characteristics of a metamaterial-based parallel-plate ridge gap waveguide realized by bed of nails," IEEE Transactions on Antennas and Propagation, vol. 59, no. 3, pp. 904-913, 2011.

[4] P.-S. Kildal, A. U. Zaman, E. Rajo-Iglesias, E. Alfonso, and A. ValeroNogueira, "Design and experimental verification of ridge gap waveguide in bed of nails for parallel-plate mode suppression," IET Microwaves, Antennas \& Propagation, vol. 5, no. 3, pp. 262-270, 2011.

[5] E. Alfonso, M. Baquero, P.-S. Kildal, A. Valero-Nogueira, E. RajoIglesias, and J. Herranz, "Design of microwave circuits in ridge-gap waveguide technology," in IEEE MTT-S International Microwave Symposium Digest (MTT). IEEE, 2010, pp. 1544-1547.

[6] A. Valero-Nogueira, M. Baquero, J. I. Herranz, J. Domenech, E. Alfonso, and A. Vila, "Gap waveguides using a suspended strip on a bed of nails," IEEE Antennas and Wireless Propagation Letters, vol. 10, pp. 1006-1009, 2011.

[7] C. Gahete Arias, M. Baquero Escudero, A. Valero Nogueira, and A. Vila Jimenez, "Test-fixture for suspended-strip gap-waveguide technology on ka-band," IEEE Microwave and Wireless Components Letters, vol. 23, no. 6, pp. 321-323, 2013.

[8] D. Sanchez-Escuderos, M. Ferrando-Bataller, M. Baquero-Escudero, and J. I. Herranz, "Reconfigurable slot-array antenna with RF-MEMS," IEEE Antennas and Wireless Propagation Letters, vol. 10, pp. 721-725, 2011.

[9] A. Corporation, "HFSS (high frequency structural simulator)," 2010, Suite v13, Pittsburg (PA), USA. 